\title{
SIMON HOST COMPUTER SYSTEM REQUIREMENTS AND RECOMMENDATIONS (U)
}

by

\author{
L. J. Harpring
}

Westinghouse Savannah River Company

Savannah River Laboratory

Aiken, South Carolina 29808

A Technical Report for Publication

This paper was prepared in connection with work done under Contract No. DE-AC09-89SR18035 with the U.4 Department of Energy. By acceptance of this paper, the publisher and/or recipient acknowled the U.S. Government's right to retain a nonexclusive, royalty. free license in and to any copyrigit covering this paper, along with the right to reproduce and to authorize uthers to reproduce all or part of the copyrighted paper.

\section{DISCLAIMER}

This report was prepared as an account of work sponsored by an agency of the United States This report was prepared as an account of work sponsored by an agency of the United employess, makes any warranty, express or implied, or assumes any legal liability or responsibility for the accuracy, completeness, or usefulness of any information, apparatus, product, or process disclosed, or represents that its use would not infringe privately owned rights. Reference herein to any specific commercial product, process, or service by trade name, trademark, manufacturer, or otherwise does not necessarily constitute or imply its endorsement, recommendation, or faving by the United States Government or any agency thereof. The views and opinions of authors expressed herein do not necessarily state or reflect those of the United States Government or any agency thereof.

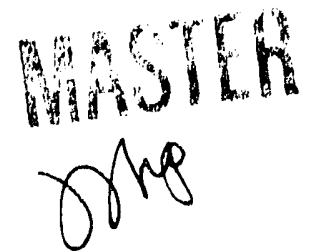




\begin{abstract}
BIMON
Bost Computar syetom

Requirements and Recommendations
\end{abstract}

\author{
L. J. Harpring \\ Electronics Development Group \\ Laboratory Bervices section \\ westinghouse savannah River Company
}

November 29,1990 


\section{General Requirements}

Development Service order \#90025 requested recommendations for computer hardware, operating systems, and software development utilities based on current and future SIMON software requirements. Since SIMON's main objective is to be dispatched on missions by an operator with little computer experience, "user friendly" haidware and software interfaces are required.

Other design criteria include: a fluid software development environment, and hardware and operating systems with minimal maintenance requirements. Also, the hardware should be expandable; extra processor boards should be easily integrated into the existing system. And finally, the use of well established standards for hardware and software should be implemented where practical.

\section{Boftware Requirements and Recommendations}

Before any part of the SIMON Host Computer system could be specified (especially hardware), software requirements for the system had to be defined.

A preliminary software block diagram was presented to the SIMON staff and, via a special meeting and information gathered from other sources, a new block diagram (Appendix A) was created. This diagram requires at least seven tasks to be running simultaneously. The only way this can be accomplished is with a multi-tasking operating system. UNIX is the most mature multi-tasking operating system and is recommended for this reason. Other requirements derived from the diagram include: multiple layers of log-in security for the operator, the system programmer, and the robotics engineer; inter-task communications; the $\mathrm{x}$ window system; and Ethernet networking (TCP/IP). UNIX is also capable of supporting all of these.

UNIX is also highly recommended for its Programmer's Workbench Software Development Tools. These include: automatic file maintenance or "make" which assures compilation of the correct version of the source code, source code Control system (SCCS) which automatically saves old versions of source code as they are created, Revision Control system (RCS) which allows for the compilation of old versions of the source code if necessary, and various debugging tools which help produce error free source code.

A hardware diagram of all SIMON operator Console inputs and outputs (Appendix B) was also created. This diagram indicates that various analog and digital signals will have to be written and read frequently at precise intervals. This will involve the use of 
interrupts. UNIX is known as a fair operating system which allocates CPU time fairly among the running tasks. No one task has priority over another. This is fine for operator interface type programs and software development, but just won't work for programs which must read or write data at precise intervals. Only a real-time operating system can do this. The real-time operating system must also support many of the features of UNIX as mentioned above. These include inter-task communications, the $x$ window System, and Ethernet network protocols. The only real-time operating system to support all of this is Vxworks from Wind River systems.

So, both UNIX and VXWorks are recommended. As described later in the Hardware Requirements and Recommendations section, each operating system will run on separate computexs yet work and communicate as if they were one. VxWorks was written with the purpose of communicating with a UNIX based computer and contains many UNIX-like features which make this relationship work smoothly. Also, VxWorks has an integrated compiler, linker and debugger which help tremendously during the software development cycle. Many real-time operating systems are table driven. This means various configuration parameters have to be determined and placed into tables in order to configure the system before a bootable ROM can be made. VxWorks comes read; with a bootable ROM to be placed into the CPU motherboard. This feature alone could save up to two month of start-up time.

There are several classes of utility software that will be helpful during software development and should be considered when making recommendations for the SIMON Host Computer system. A product called saber-c from saber software falls into the integrated programming environment utility class. It is divided into four panels or windows: the source panel, which displays source code; the options panel, used to invoke run-time and load-time commands; the message panel, which displays status and error messages; and the workspace panel, used to enter commands from the keyboard. Via these panels, the programmer can edit, compile, link, run, and debug programs without having to leave the program. This can increase productivity two to three times. The package also comes with a complete $C$ interpreter which can aid in the debugging process. Currently, saber-C is available for sun and DEC platforms, but will be available for the IBM-RS/6000 during the summer of 1991.

ExOCODE from Expert object Corporation and The Builder Xcessory from Integrated computer solutions (ICS) both allow a programmer to rapidly build prototype graphical user interfaces (GUIs) for OSF/Motif based applications. Once the programmer is satisfied with the look and feel of the GUI, both packages allow for automatic generation of $C$ source code. Later, the code can be compiled and executed to produce the exact GUI you have just built. This rapid 
prototyping will become invaluable when potential operators of the SIMON Host Computer System use various GUIs and let the software developers know what they like and dislike. Both ExOCODE and The Builder Xcessory are available for Sun, IBM and DEC platforms. The only exception is that The Builder Xcessory can only run on the CX graphics option of the DECstation 5000 model 200.

\section{Software Recommendations summary:}

- UNIX with support for

- multi-tasking

- log-in security

- inter-task communications (Sockets)

- $\quad X$ Window System (X11.4)

- Ethernet Network (TCP/IP)

- Programmer's Workbench

- Real-Time operating System (VxWorks) with support for

- multi-tasking

- interrupts

- inter-task communications (Sockets)

- $X$ Window System (X11.4)

- Ethernet Network (TCP/IP)

- Development Tools for $C$ and OSF/Motif

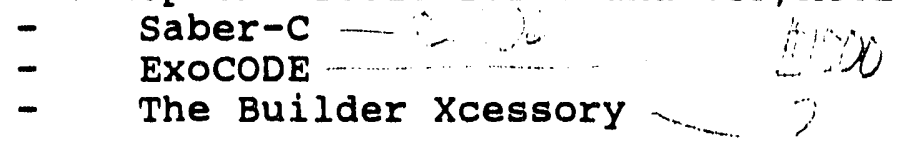

\section{Hardware Requirements and Recommendations}

Appendix $B$ indicates that at least sixteen digital inputs, two digital outputs, four analog inputs, two RS-232C ports, and one Ethernet interface (either thick or thin wire) are required. There are a plethora of computer bus standards which offer analog and digital I/O boards. In terms of availability of a wide variety of processors, digital and analog I/O, and peripheral boards, the vMEbus cannot be beat. Simply put, it is the most widely supported bus and is recommended for the above reasons.

Before this service order was issued, three VMEbus cards were ordered by EES from VME Microsystems International Corporation (VMIC). These boards include the VMIVME-1101 32-Bit Digital Input Board, the VMIVME-2170 32-Bit Optically Coupled Digital output Board, and the VMIVME-3100 16-Channel 12-Bit Analog-to-Digital Converter Board. As it turns out, these boards are adequate for all analog and digital $I / O$ as defined in Appendix $B$ and are recommended for use. 
A search for a multipurpose data acquisition board was made. This board would have contained all of the functions of the above three boards plus more. One manufacturer (Metrabyte) was found, but they no longer make or support the board. The reason for wanting a single board are less rack space and spare parts. But, it was decided that less complicated boards are less likely to fail and, if they did, they would be easier to repair. Again, this concern comes from recommending a system which will require minimal maintenance and down time.

The choice of a VMEbus microprocessor board (or target) is tied directly to the host (workstation) and target machines that Vxworks will support. As described later in this section, the IBM RS/6000 and the DECstation 5000 have been chosen as the two finalists for workstations. In order for VxWorks' development system to work correctly, software development is performed on a host. This includes cross compiling the source code to run on the target which most likely has a different processor. Once the compilation is finished, the object code is downloaded to the target processor. There are numerous combinations of host and target machines. VxWorks only supports specific combinations. For the IBM RS/6000 as the host, only the Intel 80960 RISC processor is supported as a target. For the DECstation 5000 as the host, only the MIPS R3000 processor is si pported as a target (This will officially be announced in January, 1991). This is the current state of support for the IBM and DEC machines. Support for the Motorola $680 \times 0$ processo:s will follow with an announcement in the summer of 1991.

Heurikon is the only known source of a VMEbus board with an Intel 80960 RISC processor. Even if there were more choices, Heurikon would still be highly recommended because of their good reputation. Also, VxWorks was ported to the 80960 via the Heurikon HK80/V960 single-Board Microcomputer.

Some of the Heurikon HK80/V960 board's features are 32-bit addressing, a 32-bit data path, 7 bus interrupts, an Intel $80960 \mathrm{CA}$ RISC microprocessor operating at up to $40 \mathrm{MHz}$, up to 8 MBytes of RAM, an Ethernet interface, a SCSI interface, four serial I/O ports (RS-232C or RS-422), Centronics parallel port, three 16-bit programmable counter/timer channels, and an optional time of day clock with battery backup. The only major feature the Heurikon board lacks is a math coprocessor chip. It is not expected that this will cause ary major problems. If the IBM RS/6000 is chosen as the host, then the Heurikon board is recommended for the target.

HTEC Limited, Omnibyte, and Lockheed Sanders are the only known sources of VMEbus boards with MIPS R3000 RISC processor. After finally reaching HTEC in England, it was discovered that the board is 
not being manufactured now and probably will not be in the future.

The Omnibyte OE68K/VR3000 board contains a $25 \mathrm{MHz}$ MIPS R3000 RISC processor chip set with an R3010 FPU (floating point unit), $32 \mathrm{~KB}$ of instruction cache, $32 \mathrm{~KB}$ of data cache, $8 \mathrm{MB}$ or $32 \mathrm{MB}$ of on board main memory, FIFO interrupter, one RS-232C serial port, VMEbus interrupt generator and handler, and a private memory bus. The major omissions from this board are an Ethernet port, a second RS232 port and a real time clock. Omnibyte claims the next version of this board (to be released during 1991) will also have an Ethernet port, two RS232 ports, a SCSI port and maybe a real time clock.

Lockheed Sanders produces the STAR MVP board which contains a MIPS R3000 RISC processor, an R3010 floating point unit, 8MB or 16MB of main memory, $128 \mathrm{~KB}$ data cache, $128 \mathrm{~KB}$ instruction cache, $512 \mathrm{~KB}$ EPROM, 128KB EEPROM, VMEbus interface, and two RS-232/422/423 ports. The major omissions from this board are an Ethernet port, and a real time clock. The STAR MVPs main claim to fame is that wind River Systems ported VxWorks over to the R3000 processor using the STAR MVP. Because of the VxWorks edge and the extra RS-232 port, this board is recommended over the omnibyte board. In either case, a separate Ethernet board, such as the Motorola MVME374, will have to be purchased to accompany one of the R3000 based board.

When VxWorks supports $680 \times 0$ boards as targets for IBM and DEC, the Motorola MVME147S Monoboard Microcomputer will be recommended. This board contains a 68030 Microprocessor, 68882 Floating Point Coprocessor, up to 32 megabytes of RAM, one SCSI Bus Interface, four 16-bit ROM/PROM/EPROM/EEPROM sockets, two 16-bit timers, a watchdog Timer, four RS-232 Ports, a Centronics printer port, an Ethernet interface, a VMEbus Master/Slave Interface, and more. The combination of this board and the VMIC boards will fulfill all of the real-time hardware needs as laid out in Appendix B. Also, this board is being used by other groups at SRS, namely EES, and can be maintained by on site equipment currently being set up in 100-C. The $32 \mathrm{MHz}$ version is recommended to handle the added overhead of the $\mathrm{X}$ Window system. This board is highly recommended for the above reasons.

The last vMEbus board is optional. Currently, the antenna positioning sortware is communicating via RS-232C with a Compumotor 372 Indexer. But, EES has in its possession a Compumotor 1830 Indexer which has a VMEbus interface. In general, communications over the VMEbus will be much faster than over an RS-232C port. Because of the higher speed communications and possibly easier programming, it is recommended that the 1830 board be used.

An enclosure and power supply will also be required to house the VMEbus boards. Once the target processor board vendor is chosen, 
that vendor's enclosure should be purchased. If they do not offer such an enclosure, then a vendor such as zero corporation should be chosen since they have an excellent selection of rack mountable enclosures, back planes and power supplies. system.

Figure 1 shows the basic components of the recommended VMEbus

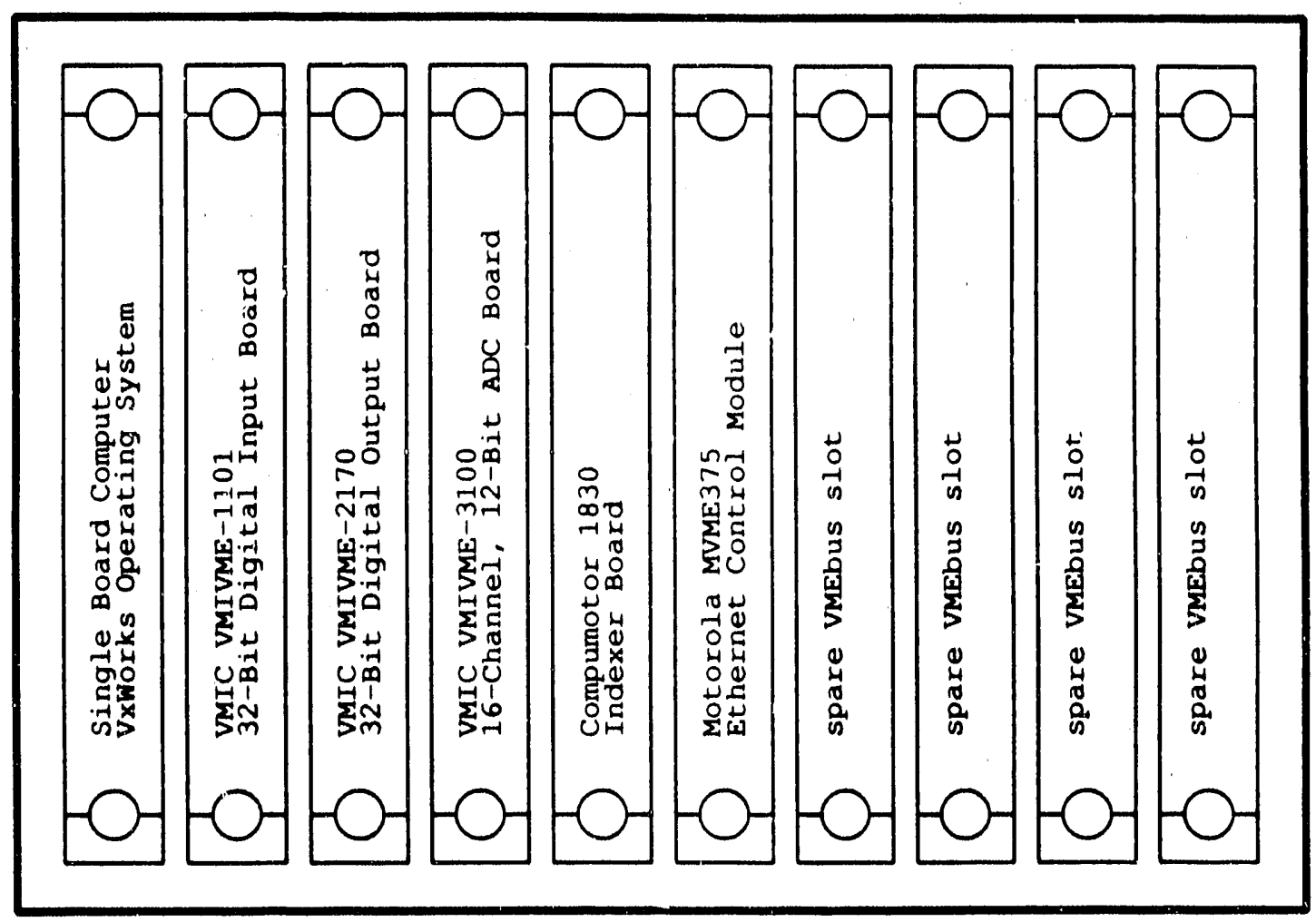

Plgure 1. VMEbus systom Diagraa

As mentioned previously, there will be two computer systems and two operating systems. VxWorks will run on the vMEbus system and UNIX will run on a workstation. There are several key criteria that must be met by a workstation. They include: the ability to run the VxWorks development software which includes a compiler, linker and debugger; run the $Y$. Window System $11.4 ;$ support software which will produce the OSF/Motif "look and feel"; support a 19 " monitor (Sony Trinitron preferred) with $1280 \times 1024$ resolution, $.31 \mathrm{~mm}$ (or smaller) dot pitch, and display 256 colors out of a palette of 16.7 million; have an Ethernet connection (thick and thin); have optional RS-232 and Centronics ports; have a 320 mbyte hard disk drive with room to add one more 320 mbyte hard disk drive; have a 1/4" tape drive; have a 
$C D$ drive; have an Ethernet printer (color optional); and run at the UNIX benchmark speed of at least 15 SPECmarks.

The SPEC benchmark or SPECmark is calculated by an independent service called The Systems Performance Evaluation Cooperative or SPEC for short. This nonprofit consortium was started by EE Times, sun Microsystems, MIPS Computer Systems, Hewlett-Packard and Apollo. The current membership also includes AT\&T, Bull, Compaq, Control Data Corp., Data General, Digital Equipment Corp., DuPont, Fujitsu, IBM, Intel, Intergraph, Motorola, NCR, Prime Computer, siemens AG, Silicon Graphics, Solbourne, Stardent, and Unisys. Part of DOD (an Air Force group) adopted the SPECmark as a standard measurement for vendor bidding on the Tactical Air Force workstation project. With industry and government support, the SPEC benchmark has been accepted as the most reliable means of measuring workstation performance. Because of this, the SPEC benchmark will be used in this recommendation.

Several workstations have been looked at including the Apple Macintosh IIfX, the DECstation 5000 Model 200 , the IBM RS/6000 Model 320 , the sun SPARCstation 1 , and the newly announced SPARCstation 2.

The Macintosh IIfX running A/UX (Apple's UNIX) is relatively slow as compared to other workstations. This information comes from a user in scientific computations section. It would be too slow for practical software development in an $\mathrm{X}$ Hindow system envirorment and even slower when trying to run applications using the $X$ window system environment. Also, the VxWorks development tools are not supported on the Macintosh IIfx.

The sun SPARCstation 1 runs at about 8.4 SPECmarks which does not leave much reserve computing power for future expansion. Bob Pettus of USC agrees the sun is not powerful enough for the SIMON project even though USC developed the SIMON software on a Sun SRARCstation 1+. Also, the SPARCstation does not directly support OSF/Motif (it supports OPEN LOOK) or OSF/Motif GUI builder programs.

The Sun SPARCstation 2 was announced November 13, 1990. Even though this machine is rated at a SPECmark of 21 , most of the speed increase is in the graphics area. So, for an application which is not graphics intensive, the SPARCstation 2 will not perform much better than the SPARCstation 1+. Even if the SPARCstation 2 did perform better in all areas, its hefty price tag knocks it out of the race when it comes to price/performance ratio as compared to the DEC and IBM machines.

Both the DECstation 5000 Model 200 and the TRM RS/6000 Model 320 meet all of the workstation requirements listed above. The only exception is that in september, 1990 IBM was only supporting X11.3, but, they will be supporting X11.4 the first quarter of 1991. The 
DEC and IBM machines run at 18.5 and 22.3 SPECmarks respectively. They both support OSF/Motif and have pledged to migrate from their current flavors of UNIX (ULTRIX and AIX respectively) to OSF/1. OSF/1, released October 23, 1990, is the first release of the Open Software Foundation's UNIX operating system. Also, OSF/Motif is an open Software Foundation standard. Sincs the DEC and IBM workstations are so close in specifications, a bid package will have to be written and the vendor with the best price/performance ratio should be chosen.

Figure 2 shows the basic components of the recommended workstation.
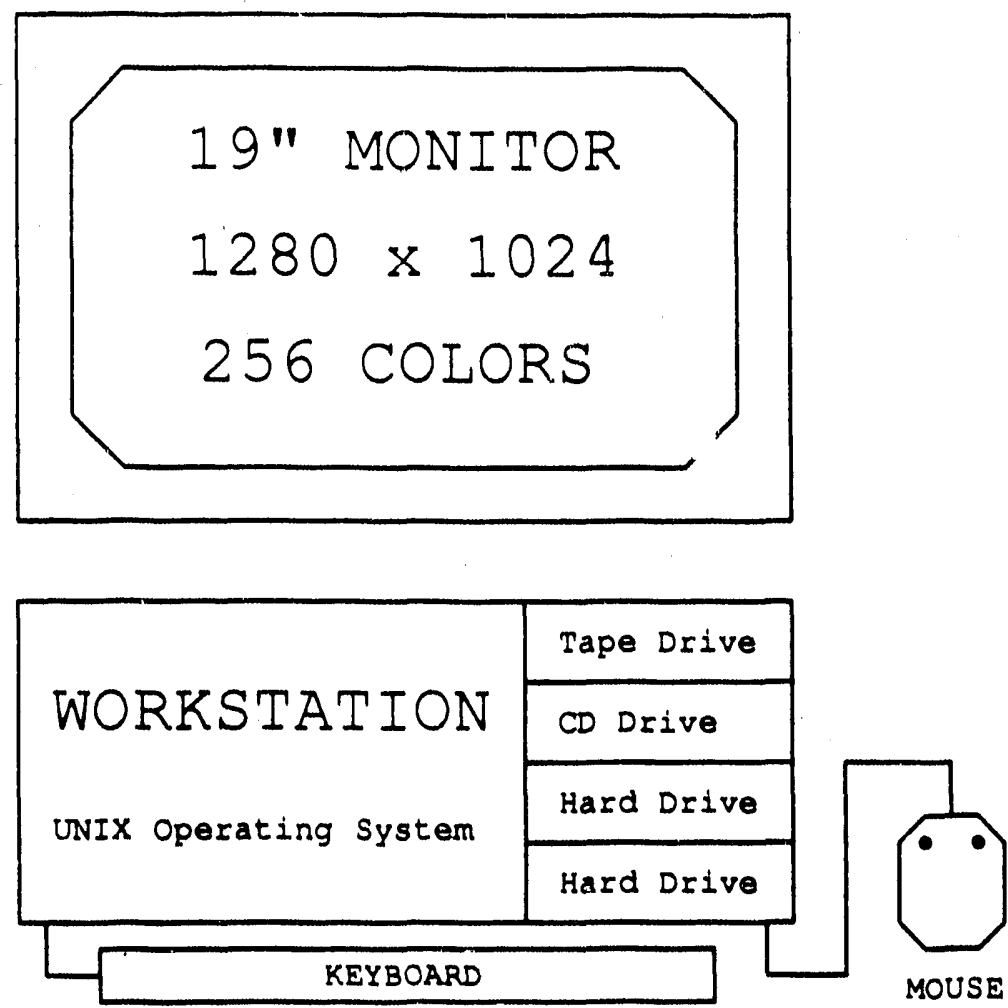

\section{Figure 2. Workstation Diagran}

An $\mathrm{X}$ terminal is needed to complete the hardware for the SIMON Host Computer system. An $X$ terminal is required for a couple of reasons. First, the SIMON staff decided that two monitors would be required to make up the system. One for the user interface which is part of the workstation, and one for a display of the world map in which SIMON will be navigating. Since the UNIX workstation and the VxWorks VMEbus computer will be communicating via Ethernet and the 
VMEbus computer does not have any graphics hardware built in, the logical choice was to tap into the Ethernet and place an $x$ terminal in line. This $x$ terminal will primarily receive $x$ window commands from the vMEbus computer, but it can also simultaneously receive $X$ Window commands from any other computer on the Ethernet cable. The $x$ Window commands originating from the VMEbus computer will be graphical representations of SIMON and his world.

There a:e a few things to consider before pursuing the $x$ terminal route. It is possible to just buy another workstation instead of the $x$ terminal. This would cost about twice as much and probably not have as good of performance, but the workstation would be available for software development or other network independent work. The $x$ termina: can still be used for software development, but it must be done over a network connected to another workstation. No $x$ terminal has virtual memory (local hard disk space) available to it, so all applications running on it must be able to fit into its memory. considering the above, the $x$ terminal is still recommended.

Specification for an $x$ terminal include: Xbench benchmark of at least 30,000 xstones; support $\times 11.4$; a monitor equivalent in resolution to the UNIX workstation (1280 x 1024,256 colors, 19 ", Sony Trinitron); enough video memory for two screens ( 2.5 Mbytes), enough system memory for $X$ server code and sufficient fonts and expandable to 8 Mbytes; keyboard; mouse; thick and/or thin wire Ethernet; setup screens for the monitor; and support the host workstation chosen.

optional but desirable features inclide: all X server code stored locally in ROM, fonts stored in ROM, password protection on all setup menus, non-volatile setup menu memory, RS-232 port, Centronics port, "backing-store" feature which saves locally whatever is about to be covered by a new window, and "save-under" which saves locally what is temporarily covered by a pop-up menu or other graphic element.

The standards for measuring the performance of $X$ terminals are not nearly as widely accepted as those for the performance of workstations. There are currently no recognized benchmarks for measuring network performance of $x$ terminals, but there are two leading benchmarks that measure the display performance of $x$ terminals: "xbench" and "xilperf". The x11perf software has been denounced for producing too many results which cannot be generalized (i.e. they are not fair to all X terminals). Xbench produces a single number which fails to distinguish between the hardware and software performance of the $x$ terminal, but it is the best known $x$ terminal benchmark and will be used in this recommendation. The xbench benchmark produces a value called the "xstone". A value of 10,000 xstones is the value that a Sun-3/50 produces over UNIX sockets. A socket is a formal protocol used to communicate between 
tasks over a network.

The October 1990 issue of UNIXWORLD features an article entitled "X TERMINALS PUT TO THE TEST". Author BOb Lemon and the UNIXWORLD staff tested $x$ terminals of several vendors using a modified xbench benchmark. These modification made xbench more fair and resulted in producing three separate categories of xstones: Software Developer, Desktop Publisher, and CAD/CAM. The CAD/CAM results were used for our evaluation since they are more closely related to the graphics of SIMON and his world.

The top five performers in the CAD/CAM category were: Datacube XS16-1, 86,728 xstones; Human Designed Systems (HDS) V191, 50,569 xstones; Network Computing Devices (NCD) NCD16e, 48,675 xstones; Princeton Graphics Systems Ultra XC-30, 47,947 xstones; and Visual Technology X-15SR, 46,656 xstones. In terms of price/performance ratio in units of dollars/xstone, the top five were: Visual, $\$ 0.06$; Datacube, $\$ 0.06$; HDS, \$0.07; NCD, \$0.07; and Princeton, \$0.08.

The above $X$ terminals are not the exact models that would meet the specifications of the requirements. From looking at data sheets, the following company's models do meet the requirements: Visual Xbase/12, HDS V1.9C8, and Tektronics XP29. The IBM Xstation 120 and the DEC VT1300 both missed the mark by only supporting X11.3, but they will probably support $X 11.4$ sometime next year. No data sheets have been received from Princeton Graphics or Datacube to determine if they have an adequate model.

of the $X$ terminal vendors who have sent data sheets, the visual $\mathrm{Xbase} / 12$ is the leader. It meets all of the mandatory and optional specifications except for not having an option for a Centronics port. The most unique feature of the $\mathrm{xbase} / 12$ is that it does not come with a monitor, and the mouse and keyboard are optional. This allows the buyer to combine the Xbase/12 with any monitor from Visual's approved list (which is very exhaustive). This would allow for two identical monitors to be used in the sIMON Host computer System console which would be more aesthetically pleasing. For the above reasons, the Visual Xbase/12 is recommended in combination with a monitor, mouse, and keyboard.

\section{Hardware Recomendations summary:}

- VMEbus Microprocessor Cards

- If IBM is the host, use the
0 Heurikon HK80/V960

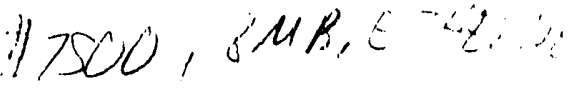

- If DEC is the host, use one of the following:

- Lockheed Sanders STAR MVP

Page 10

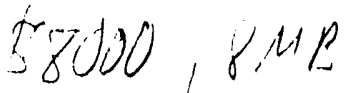


- Omnibyte OB68K/VR3000

$\because \hat{H}$

If VxWorks support's the $680 \times 0$ for the IBM or DEC, use - Motorola MVME147S

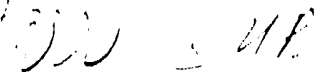

- VMEbus Expansion Cards

- VME Microsystems International VMIVME-1101, 32-bit TTL Digital Input Board

- VME Microsystems International VMIVME-2170, 32-bit optically Coupled Digital Output Board

- VME Microsystems International VMIVME-3100, 16-Channel 12-Bit Analog-to-Digital Converter Board

- Compumotor 1830 Indexer

- Motorola MVMr374 Ethernet Control Module -

- UNIX Workstation Specifications

- Must run VxWorks development tools (compiler, linker, debugger)

- support the $X$ window System 11.4

- support OSF/Motif

- 19" monitor with .31mm (or smaller) dot pitch

- $1280 \times 1024$ resolution

- 256 colors (minimum for color X windows)

- thick and thin wire Ethernet

- SPECmark benchmark of at least 15

- optional RS-232C and Centronics port

- X Terminal specifications

- Xbench benchmark of at least 30,000 xstones

- support the $X$ Window Syltitem 11.4

- monitor equivalent to GRIX workstation

- 2 screens of video memory (2.5 Mbytes)

- enough system memory for $X$ server code and downloadei fonts and expandable to 8 Mbytes

- keyboard

- mouse

- Ethernet

- setup screen for monitor

- support the host workstation chosen

- Optional $x$ Terminal specifications

- $X$ server code stored locally in ROM

- fonts stored locally in ROM

- password protection on all setup menus

- non-volatile setup menu RAM

- RS-232C

- Centronics Port

- backing-store

- save-under

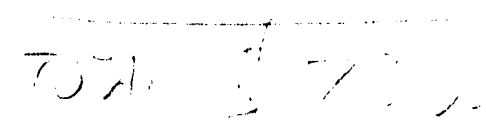

Page 11 


\section{Result of Requirements: A Distributed processing system}

The software and hardware requirements led to the recommendation of a distributed processing system. This means that computer programs or tasks are executed in parallel on separate processors. One of the advantages of this architecture is more processing in less time. But, the driving force behind the architecture is the need for two distinct types of operating systems as described in the software Requirements and Recommendations section of this report.

Figure 3 shows a block diagram of the SIMON Host Computer system.

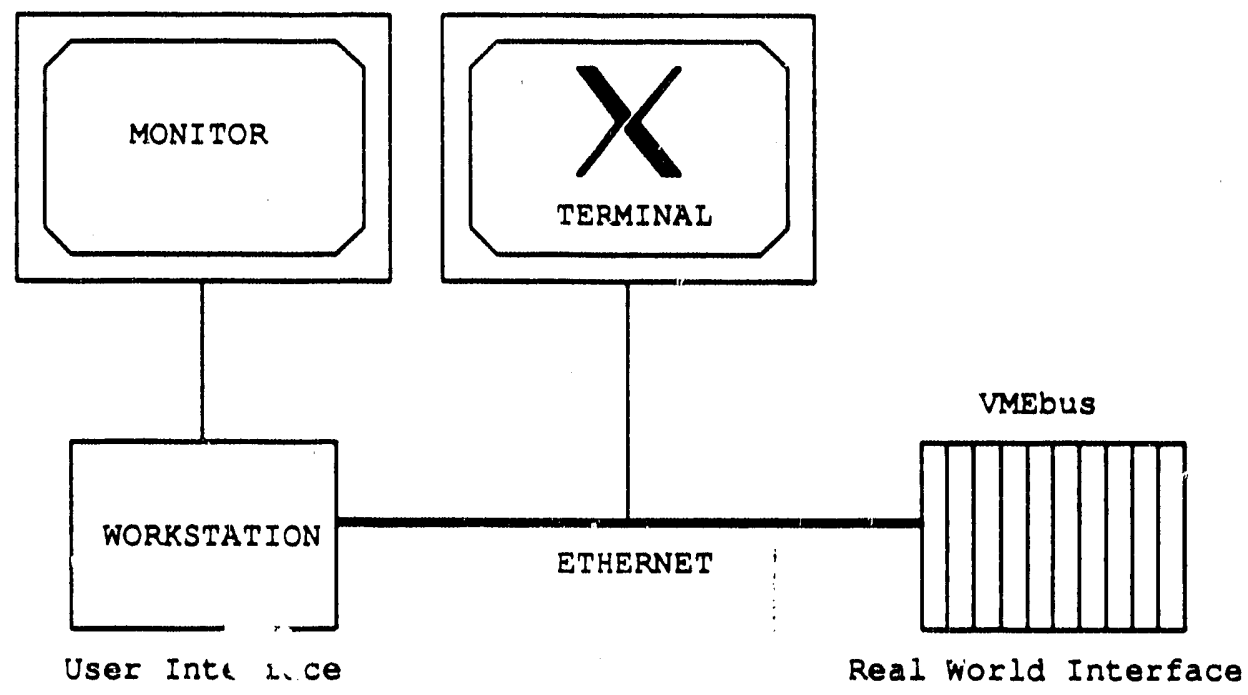

Figure 3. System Diagram

\section{Expandable Hardware and software}

The recommended computer architecture of the SIMON Host Computer system is extremely expandable in terms of hardware and software. More tasks can be added to the workstation or the VMEbus CPU if more proce ssing is required. If the new tasks decrease system performance too wuch, one or more CPU voards can be added to the VMEbus. Existing tasks can be moved to the new CPUs without coding changes. This is possible only if UNIX sockets are used for communications between tasks as outlined in the VxWorks manuals.

Complete subsystems may be added to this architecture without worry of extensive (if any at all) coding changes. Subsystems would include: more VMEbus backplanes for remote locations; more $\mathrm{X}$ 
terminals for added system monitoring or software development capabilities; or even more workstations for software development or what ever need may arise.

The above expansions are possible due to the computer system's architecture. More specifically, this includes the UNIX and VxWorks operating systems, and the Ethernet and the $X$ window system protocols.

\section{Maintenance and Training Requirements}

Although the SIMON Host Computer system has been designed for minimal maincenance, maintenance will still be required. In order to prepare for this, it is recommended that training for computer hardware and software maintenance personnel be included in the purchase order or bid package to the respective hardware and software companies.

As stated in Appendix C, LSS Computer Maintenance will maintain as much of the SIMON Host Computer system hardware as practical. Ir EES wants LSS to maintain a one of a kind piece of hardware, then EES will have to provide training for LSs personnel. LSS will provide their own training for the more common hardware items which are used other places in SRL. Currently, this would include the DECstation 3100, but more items will be added to this list as more employees begin to purchase workstations. Maintenance of operating systems, networks, and application software will be the responsibility of EES. 


\section{Appendix A}

8IMON Host software Block Diagram 


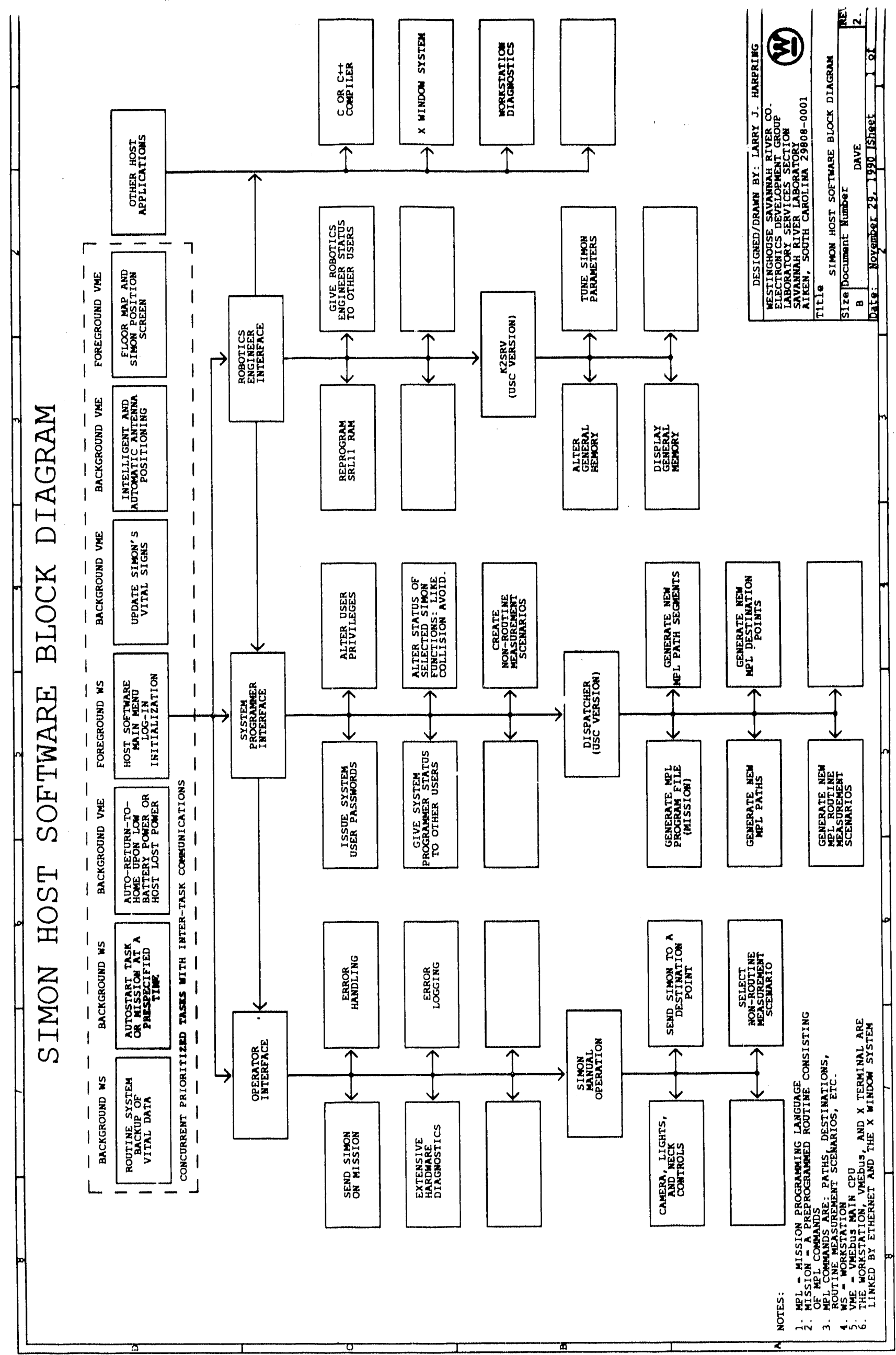


Appendix B

simon Host Computer Inputs and outputs 


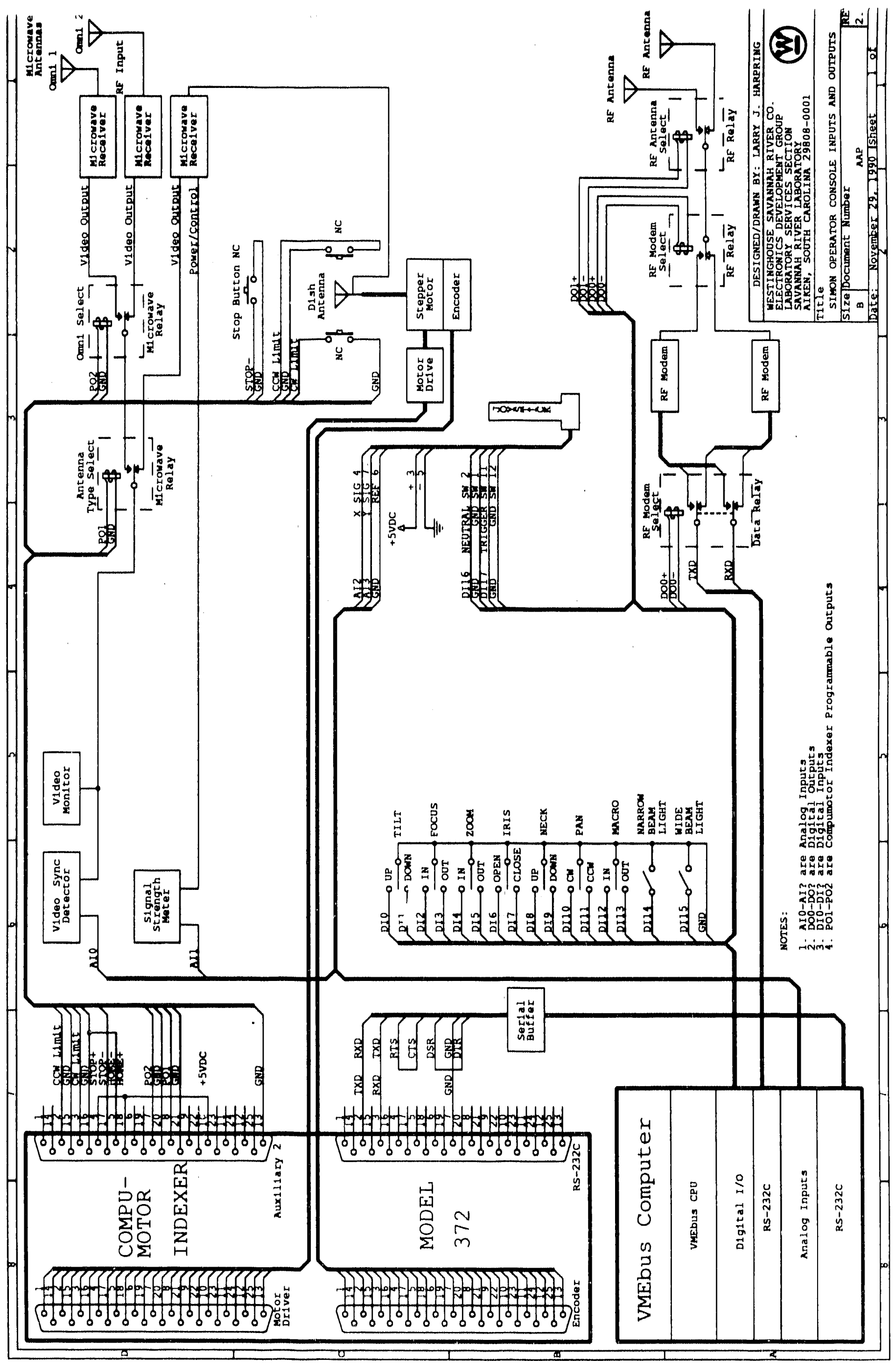


Appendix $C$

Clarification of Ls8 Assistance to the sIMON Robot Project 


\section{INTER-OFPICE MEMORANDUM}

Savannah River Site

TO: M.A. Kyle

TO: FRANK HECKENDORN

CC: DAVID W. WILKERSON

CC: I,uther E. Wilhite

$\begin{array}{ll}\text { Date: } & 31-\text { May-1990 } 08: 46 \text { am EST } \\ \text { From: } & \text { Larry J Harpring 773-A } \times 52656 \\ & \text { HARPRING-LJ-T5721 } \\ \text { Dept: } & \text { SRL/LSS } \\ \text { Tel No: } & \text { X52656 }\end{array}$

( KYLE-MA-04833)

( HECKENDORN-FM-T5735)

(WILKERSON-DW-T4944)

( WILHITE-LE-T7671)

subject: Clarification of LSS Assistance to the SIMON Robot Project

I would like to clarify the responsibilities of LSS and EES with respect tc the SIMON project. The following items are my major areas of concern. I want to mention them early enough in the process so that there will be no surprises for anyone in the future. The following lists of responsibilities are not exhaustive. LSS Development has other responsibilities as stated in the basic data for the service order. The purpose of the lists is to make sure all groups involved are aware of these particular responsibilities and prepare to handle hem as needed.

Responsibilities of LSS Development.

1) Make recommendations for the sIMON host computer system which includes hardware, operating system, development (programming) environment, development language and other associated software such as utilities.

Responsibilities of LSS Computer Maintenance:

1) Maintain portions of the host computer system hardware. Exactly which parts will be maintained is negotiable and can only be decide upon after the system recommendations have been made. When or if any of the system is moved outside of the SRL complex, LSS Computer Maintenance will no longer have authority to maintain the hardware. All computer hardware outside of the SRL Complex is the responsibility of the DC\&S group.

Responsibilities of EES Robotics:

1) Purchase the recommended host computer system. This includes preparing all necessary paper work and, if required, writing a bid package. If needed, LSS Development will be glad to offer consulting assistance in writing the bid package.

2) Arrange for training, if necessary, on the use and maintenance of the ystem. This can potentially involve several different hardware and software company training programs. It is recommended that these arrangements be made as part of the purchase order for the host system and that Lss Computer Maintenance be included in the training. 
3) Maintain the host computer software. This includes the operating system, network management, system management, and application software. LsS Development will make system recommendations which will reduce maintenance requirements as much as possible while still meeting the needs of the host computer system. No application soffware written by LSS will be turned over to EES until EES is satisfied that it is in a maintainable state. This will include documented source code, program description and explicit instructions or producing executable code from the source code.

4) Maintain host computer system hardware which Lss Computer Maintenance cannot support. 

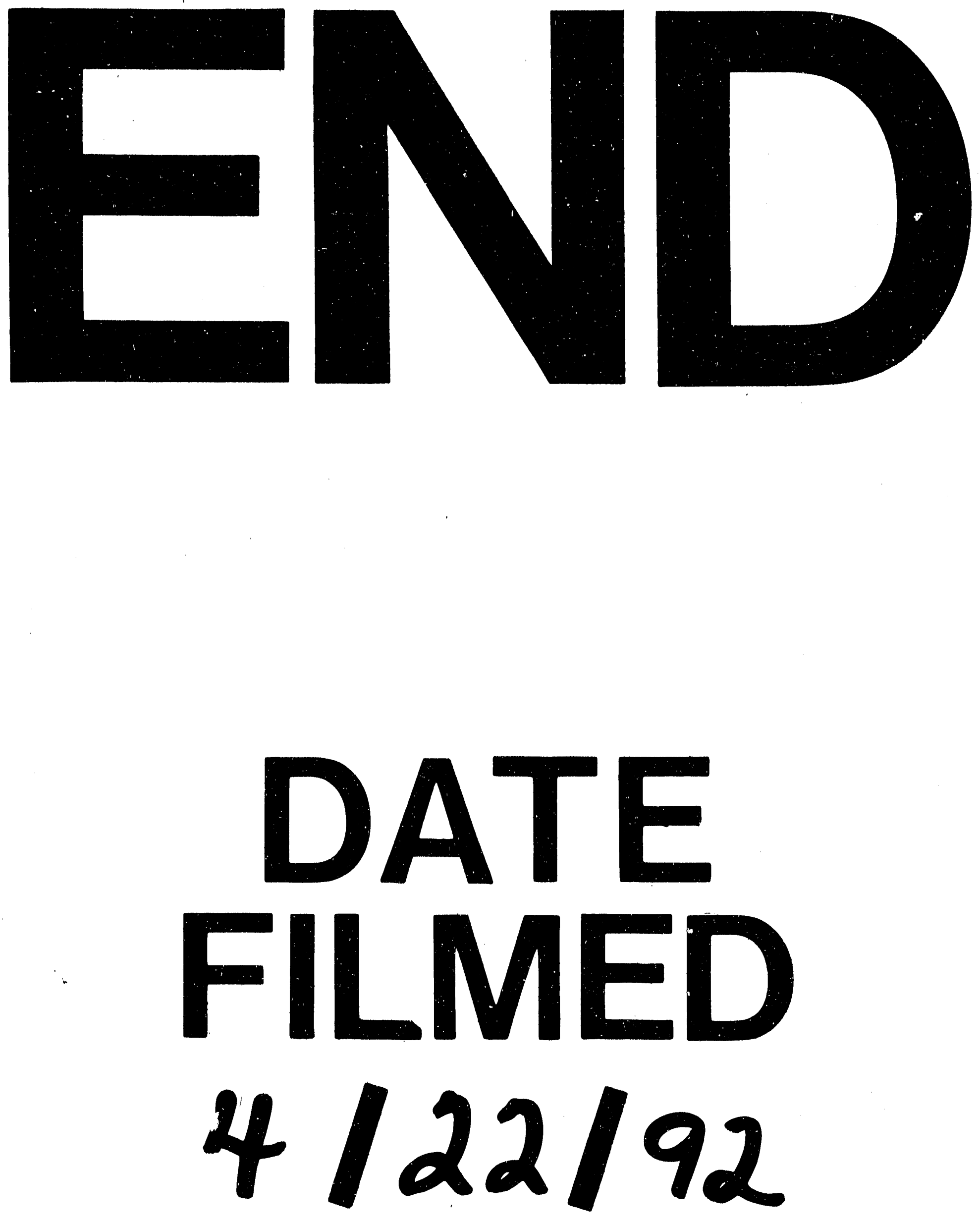

1 
2010

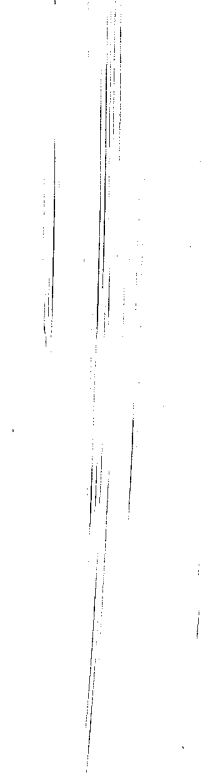

